\title{
The Major Ozonated Autohaemotherapy: Biochemical Background and a Proposed Study for an Integrated Therapy in Neurosurgical Diseases
}

\begin{abstract}
Mini Review
The so-called "major ozonated autohaemotherapy" (MOAT) was invented in Germany in the last Century and until now millions of treatments have been performed in patients all over the world without any acute or chronic toxicity. The correct method consists in collecting 100-200 ml of blood (plus an anticoagulant) in an ozone resistant glass bottle, adding an equivalent oxygen ozone gas volume containing ozone at a precise concentration ranged from 10 to 40 micrograms per milliliter of gas mixture, gently mixing for $2 \mathrm{~min}$ and returning the oxygenated-ozonated blood to the donor during the next 15 min, obviously without the gas. A complete description of the methodology was reported in previous articles [1-2].
\end{abstract}

When human blood is exposed to a gas mixture composed of medical oxygen and ozone (about $96 \%$ and $4 \%$ respectively), both gas present in the phase overlying a superticial layer of about $10 \mu$ of blood at first dissolve in the water of plasma. The gas solubilization goes on continuously when the blood is gently rotated in a glass bottle. Oxygen equilibrates with the extracellular and the intraerythrocytic water before becoming bound to hemoglobin until it is fully oxygenated, as shown by the rapid increase of the p02 from about 40 up to $400 \mathrm{mmHg}$. On the contrary ozone, more soluble than oxygen, readily dissolves in water and reacts instantaneously with several substrates, oxidizing ascorbic acid, $u$ rate, free cysteine, glutathione (GSH) molecules and albumin thiol groups (SH). In fact ozone doses within the therapeutical range (10-40 $\mu \mathrm{g} /$ $\mathrm{ml}$ of gas with a volumetric 1:1 gas- blood ratio) are mostly neutralized by the well known sacrificial reactions: for example albumin is considered the main sacrificial molecule due to large amount in plasma and surely it prevents lipoprotein damage.

The ozonation process in blood is therefore characterized by the formation of only few molecules of reactive oxygen species (ROS) and lipid oxidation products (LOP) acting in two phases : while ROS ( mainly hydrogen peroxide) are acting immediately and disappear (early and short-acting messengers), LOPs (late and long-lasting messengers), via the circulation, distribute throughout the tissues and either bind to cell receptors or enter into the cell.

The behavior and pharmacodynamic of hydrogen peroxide have been ascertained: the initial formation of a gradient between plasma and intracellular water allows its entrance into the erythrocytes and lymphocytes but its concentration remains around a few micromoles because it is quickly reduced to water by free glutathione, catalase and glutathione-peroxidase. Its half-life is less than one second

Molume 8 Issue 1 - 2017
Emma Borrelli*
Department of Medical Biotechnologies, University of Siena,
Italy
*Corresponding author: Emma Borrelli, Department of
Medical Biotechnologies, University of Siena, Italy, Email:
borrelli@unisi.it
Received: July 09, 2017 | Published: July 24, 2017

and yet its intracellular concentration is critical because, in order to activate some biochemical pathways (formation of oxidized glutathione with consequent activation of the pentose cycle in the red cell and activation of a tyrosine kinase in lymphocytes), it must reach a critical threshold that nonetheless, is not cytotoxic. Provided that the ozone dose is within a well defined, experimentally determined range (10$40 \mu \mathrm{g} / \mathrm{ml}$ or 0.21 and 0.84 micro Moles per $\mathrm{ml}$ of blood) , there is only a transitory decrease (no more than $25 \%$ ) of the potent antioxidant capacity of plasma, fully reconstituted within $20 \mathrm{~min}$ owing to the efficiency of the redox system. There is neither damage to erythrocytes: haemolysis is negligible (from $0.4 \%$ up to $1.2 \%$ ) and methemoglobin remains normal, nor to other blood cells. It must be added that ozonated erythrocytes show an improved glycolysis with an increase of ATP and 2, 3 DPG levels, which are able to shift the dissociation curve of oxyhemoglobin to the right, confirming the observation of an improved delivery of oxygen in peripheral obstructive arterial disease. The beneficial physiological cellular use of ROS is now being demonstrated in different field, including intracellular signals and redox regulation. It has been documented that low levels of ROS are signaling molecules, modulating cell proliferation and apoptosis, gene expression through activation of transcription factors like nuclear factor kappa beta (Nfk $\beta$ ) and hypoxia inducible factor (HIF).

What happens during the reinfusion of the hyperoxygenated-ozonated blood into the donor?

The hyperoxygenation of blood $\left(\mathrm{pO}_{2}\right.$ about $\left.400 \mathrm{mmHg}\right)$ is irrelevant because, during the $15 \mathrm{~min}$ infusion period, it mixes with about 6 liters of blood so that the final venous $\mathrm{pO}_{2}$ relative pressure is hardly modified. LOPs (mainly 4-hydroxynonhenale), as already mentioned, disappear from the circulation within a few minutes, 
and yet they can exert stimulatory effects throughout the body without toxicity because their concentration, at a submicromolar level, is transitory. This is a crucial consideration to keep in mind and emphasizes how a small and precise ozone dose can act as a biological response modifier. The atoxicity of blood ozonation is explained by the use of small and well calibrated doses of ozone that are tamed by the antioxidant system and the short span (only a few minutes) of ozone exposure.

The repetition of ozonated autohemotherapies in patients upregulates the synthesis of several antioxidant enzymes (Superoxide dismutase, GSH-Peroxidase, GSH-Reductase, and G6PD) and heme-oxygenase-1 (HO-1) which is one of the most protective enzymes catalyzing the release of useful compounds such as bilirubin and $\mathrm{CO}$ from heme. The trace of hemolysis $(0.4$ $-1,2 \%$ ), unavoidable when blood is ozonated in a glass bottle, is useful because it acts as an inducer of HO-1. Thus, a small, acute stress on blood up regulates the antioxidant defences and the term "hormesis" describes these beneficial effects after an exposure to a low-intensity stressor. Whether ROS will act as damaging, protective or signaling factors depends on the delicate equilibrium between ROS production and scavenging at the proper time and side.

On the basis of the ability of MOAT to restore the antioxidant potential in condition of chronic oxidative stress we integrated the ozone therapy in the treatment of patients affected by three serious and diffuse chronic oxidative stress diseases, the chronic obstructive pulmonary disease (COPD), the age related macular degeneration (ARMD), and the chronic peripheral ulcers. The results reported in published pilot studies suggested an efficacy of MOAT and a favorable cost benefit ratio [3-5].

\section{Is MOAT useful in the integrated treatment of disc herniation and low back pain?}

As previous reported, the use of MOAT in the treatment of the chronic oxidative stress diseases appears promising and patients reported a subjective and objective benefit. In patients with neurosurgical disorders the pain has a multifactorial origin and the ozone can surprisingly displays a number of beneficial effects ranging from the inhibition of inflammation, correction of ischemia and venous stasis and finally an induction of a reflex therapy effect by stimulating anti-nociceptor analgesic mechanisms [6]. On this basis we wondered if in these patients the associated treatment with local ozone injection and major ozonated autohaemotherapy could be more effective on the symptomatology respect to a simple local gas administration.

At this purpose we recently performed a pilot clinical study on 40 male and homogeneous patients with disc herniation (unpublished data). All patients between 18 and 70 years of age with sciatica since 6-8 weeks are eligible for inclusion in the study. A disc herniation at the appropriate level was assessed by Magnetic Resonance Imaging. Patients were enrolled in the study from February to November 2014. All subjects received one intradiscal injection of gas and eight major ozonated autohaemotherapy two times a week $(150 \mathrm{ml}$ of blood $+150 \mathrm{ml}$ of ozone at a increasing concentration of 20-30 micrograms $/ \mathrm{ml}$ of gas according to Borrelli et al.). Before and after the last treatment of MOAT we analyzed in all patients the plasma values of Reactive Oxygen Metabolites (d-ROM test, Diacron International, Grosseto, Italy) and the SH proteins (Diacron International, Grosseto, Italy) to better understand the time course of the oxidative stress and the antioxidant balance. The patients showed a marked increase in ROM value ( $480 \pm 40$ UCARR) and a decrease in protein SH content $(300 \pm 50$ micromols $/ \mathrm{L})$ in plasma before the treatment. After the associated treatments intradiscal ozone + MOAT a decrease in d-ROM (320 \pm 54 UCARR) and an increase of protein SH $(480 \pm 67$ micromols/L) were observed. Moreover, we found a correlation between reduced plasma oxidation values and improvement of symptoms. A large study with the measures of more accurate markers of plasma lipid peroxidation and their correlation with the patient's symptomatology is under way [7].

Nevertheless these preliminary results suggest a beneficial action of the combined treatments (intradiscal plus MOAT) in the reduction of low back pain and disability following disc herniation. We think that especially in patients with a long history of chronic pain or neurodegenerative diseases, combined treatment intradiscal/MOAT appears able to reduce the simptomatology and to restore an antioxidant balance with stable results in the follow up. More clinical studies on this issue are mandatory

\section{Other Applications of MOAT in neurosurgery}

Surgical interventions (especially in neurological diseases) represent a well known situation of oxidative, immunological and inflammatory stress for the patients. In this case the use of MOAT should be effective for a reduction of the oxidative and inflammatory damage and for a more rapid recovery of the patients after surgery.

We are planning a cycle of 4-6 MOAT at low concentrations (20micrograms $/ \mathrm{ml}$ ) before and after neurosurgical interventions for the improvement of the outcome: the primary endpoints will be the postoperative reduced risk of infections and the reduction of the recovery time through the increase of blood antioxidant levels and the decrease of the surgical immunosuppression. On the basis of the previous experimental works we suppose that this short time should be able to elicite an immunological and antioxidant response in the patients.

Moreover, a protocol about the use of intraoperative ozonated water and its wonderful antiseptic power is in progress.

\section{Conclusions}

On the basis of biochemical and clinical studies performed in the last two decades, it has become clear that ozone, in very small dosages, behaves as a real drug and the biochemical and molecular mechanism of action are well within orthodox medicine. The efficacy of the MOAT for restoring antioxidant balance is well ascertained. Patients with neurosurgical diseases could benefit for combined treatments of local and systemic ozone therapy with a better outcome and reduced expenses but unexplainably the World Health Authorities, always concerned with the increasing medical costs, still refuse to evaluate the ozone therapy and recognize its validity. 


\section{References}

1. Bocci V, Borrelli E, Travagli V, Zanardi I (2009) The ozone paradox: ozone is a strong oxidant as well as a medical drug. Med Res Rev 29(4): 646-82

2. Bocci VA (2006) Scientific and medical aspects of ozone therapy. Arch Med Res 37(4): 425-435.

3. Borrelli E, Diadori A, Zalaffi A, Bocci V (2012) Effects of major autohemotherapy in the treatment of dry age related macular degeneration: a randomized controlled clinical study. Int J Ophthalmol 5: 708-713.

4. Borrelli E, Bocci V (2014) Oxygen ozone therapy in the treatment of chronic obstructive pulmonary disease: An integrative approach. American Journal of Clinical and Experimental Medicine 2(2): 9-13.
5. Borrelli E, De Monte A, Bocci V (2015) Oxygen Ozone Therapy in the Integrated Treatment of Chronic ulcer: a case Series Report. International Journal of Recent Scientific Research 6(5): 4132-4136.

6. Borrelli E (2011) Mechanism of action of oxygen ozone therapy in the treatment of disc herniation and low back pain. Acta Neurochir Suppl 108: 123-125.

7. Borrelli E, Alexandre A, Iliakis E, Alexandre A, Bocci V et al. (2015) Disc Herniation and Knee Arthritis as Chronic Oxidative Stress Diseases: The Therapeutic Role of Oxygen Ozone Therapy. J Arthritis 4: 161 . 